\title{
Alternativas logísticas para el transporte de granos
}

( Ezequiel Ayala

Universidad Tecnológica Nacional, Argentina.

\author{
Darío N. Federman \\ Universidad Nacional de Quilmes, Argentina.
}

\author{
Juan F. Mangiameli \\ Universidad Provincial de Ezeiza, Argentina.
}

\author{
Federico Marcó \\ Universidad Nacional Arturo Jauretche, Argentina.
}

Recibido: 24 de octubre de 2018. Aceptado: 19 de junio de 2019.

\section{Resumen}

Este trabajo se enfoca en describir el transporte de cereales, oleaginosas y sus derivados dentro de la República Argentina, sector económico en el cuál nuestro país es competitivo a nivel internacional. Sin embargo, dada la extensión territorial del país, existe una importante disparidad en función de la distancia al puerto de cada punto de producción. Estas distancias disímiles generan desventajas en la ecuación económica debido principalmente al elevado costo del flete automotor y los menores rindes por hectárea. Se hará foco entonces en los distintos modos de transporte y su relevancia en mantener competitivos los costos logísticos de aquellos productos que deben atravesar grandes distancias desde sus lugares de producción a los centros de consumo o exportación. Para interpretar este fenómeno multidimensional se elabora un diagnóstico de la situación general del transporte ferroviario y de las concesiones más especializadas en transporte de granos. A partir de este diagnóstico se proponen medidas de corto y mediano plazo para mejorar el servicio y se evalúa la alternativa fluvial en caso de que el transporte ferroviario no sea conveniente.

\section{Palabras clave}

\section{Logística}

Transporte de Carga

Costos

Competitividad

Producción Agrícola

\section{Palavras-chave}

Logística

Transporte de mercadorias Custos

Competitividade

Produção agrícola 


\section{Logistics alternatives for grains transport}

\begin{abstract}
This work focuses on describing the internal transport of cereals, oilseeds and their derivatives, economic sector which in our country is internationally competitive. However, given the important geographical extension of Argentina, there is an important disparity depending on the distance to the port of each place of production. These different distances generate disadvantages on economic equation mainly due to the high cost of freight and the minor render per hectare. The focus will be on the different modes of transport and relevance in maintaining competitive the logistic costs of those products that must travel long distances from their production sites to the centers of consumption or export. To interpret this multidimensional phenomenon, a diagnosis of the general situation of rail transport and the most specialized concessions in grain transport is developed. Based on this diagnosis, short- and medium-term measures to improve the service are proposed, and the river alternative is evaluated in case rail transport is not convenient.
\end{abstract}

\section{Introducción}

Este trabajo se enfoca en describir el transporte interno de cereales, oleaginosas y sus derivados. Es sabido que la producción de granos y su posterior procesamiento constituyen actividades en las cuáles nuestro país es competitivo a nivel internacional. Sin embargo, existe una importante disparidad en la ecuación económica en función de la distancia al puerto, que se origina por el elevado costo del flete automotor y los menores rindes por hectárea, tal como se destallará más adelante.

Se analizarán entonces las distintas alternativas logísticas y su relevancia en mantener competitivos los costos de aquellos productos que deben atravesar grandes distancias desde sus lugares de producción a los centros de exportación y/o consumo. A lo largo del trabajo se localizará geográficamente la producción, la cual se realiza en el interior. Luego estos granos son transportados a las principales terminales portuarias del país con destino a su posterior procesamiento y exportación. Estas terminales portuarias se encuentran a lo largo del corredor Buenos Aires-Rosario, a las cuales pueden adicionarse los puertos de Quequén y Bahía Blanca hacia el sur de la provincia de Buenos Aires.

El procesamiento de estos granos se realiza principalmente en el entorno de la ciudad de Rosario, a orillas del Río Paraná en la provincia de Santa Fe. Pero, tal como este trabajo lo abordará más adelante, existen otros puntos de procesamiento en el interior del país.

Una porción de esta producción -principalmente derivados del trigo y secundariamente aceite de girasol y aceite de maíz- se consume localmente. Pero como el consumo de estos productos se realiza en las principales ciudades, el núcleo central del trabajo consiste en analizar las alternativas para trasladar los productos desde el interior del país a los centros urbanos, ya sea para su procesamiento, exportación o consumo doméstico.

Si bien a lo largo del trabajo se localiza geográficamente la producción y se identifican demandas de carga potenciales, este trabajo no apunta a sustituir la creación de un registro de carga. De hecho, la constitución de este registro es una de las propuestas que se abordarán más adelante.

\author{
Keywords \\ Logistic \\ Freight Transport \\ Costs \\ Competitiveness \\ Agricultural Production
}


La matriz logística del transporte de granos se basa en el modo automotor a través de camiones, lo que eleva la incidencia del flete en el valor del producto transportado.

Este trabajo describe y busca desanudar una contradicción de la economía argentina actual. El sector agrícola argentino viene creciendo a tasas considerables en los últimos 20 años. Y las regiones alejadas de los puertos crecen a tasas superiores a la media nacional. Con lo cual, es razonable esperar un uso más intensivo de modos de transporte como el ferroviario y el fluvial, que reducen los costos medios y marginales a mayor volumen y mayor distancia. Sin embargo, el transporte ferroviario de granos y derivados durante los últimos años creció por debajo de las altas tasas en que lo hizo su producción y procesamiento de granos. Y tasas muy inferiores al crecimiento de la producción en el noroeste argentino (NOA) y noreste argentino (NEA). Tampoco han surgido alternativas para el transporte fluvial.

El primer paso en el trabajo es localizar geográficamente la producción de los cuatro principales granos que produce el país: soja, maíz, trigo y girasol. Se muestra la evolución en los últimos años, analizando el comportamiento por región y haciendo hincapié en algunas provincias puntuales. Se analizan también algunas medidas que afectaron al sector, como los derechos de exportación.

Todos los datos estadísticos sobre estos cuatro granos utilizados a lo largo del trabajo son tomados del Dataset de Datos Agroindustriales de la Secretaría de Agroindustria, salvo que se indique lo contrario. El período temporal inicia con la temporada 1994/1995 y finaliza con los datos de la cosecha 2016/2017.

El segundo paso es realizar un diagnóstico de la situación general del transporte ferroviario y de las concesiones vinculadas al transporte de granos y subproductos. Se analiza su evolución reciente vis a vis la evolución de la producción.

Una importante contribución de este trabajo es la creación y cálculo de un Índice de Penetración del Ferrocarril en el transporte de granos. Este indicador será explicado con detalle a lo largo del trabajo, pero se adelanta que mide cuanto incide el modo ferroviario en el transporte terrestre de granos y sus derivados.

El tercer paso consiste en analizar la matriz de origen y destino (matriz OD) para las dos principales concesiones ferroviarias vinculadas al transporte de granos y derivados. Del lado de los aportes, este trabajo contribuye mostrando el flujo de carga de granos de cada concesión. Desde el origen, acopio o procesamiento en una provincia, hasta el destino, centro de consumo o de embarque para la producción. En ese sentido, es una representación geográfica del movimiento de un sector clave en la economía argentina. Se identifican volúmenes producidos a nivel partido/departamento, infraestructura disponible y potencialidad de la carga.

Para el análisis estadístico del transporte ferroviario de los cuatro granos (y sus derivados) seleccionados, se toman los datos estadísticos publicados por Comisión Nacional de Regulación el Transporte (CNRT), salvo que se indique lo contrario. Se ha efectuado un agrupamiento, ya que la serie histórica publicada no mantiene los mismos rótulos a lo largo del tiempo.

Además, se propone una alternativa fluvial para la producción del NEA. Como se verá a lo largo de este trabajo, una porción de la producción de granos se transporta por camiones hacia los puertos de Rosario, pero podrían embarcase cerca de su lugar de producción. Para eso es necesario realizar inversiones para mejorar la infraestructura del país, buscando abaratar costos a través de modo fluvial. 
El cuarto paso consiste en analizar las regulaciones introducidas y cambios en el marco normativo, considerando que el Estado de liderar la discusión y que la misma debe ser planteada como un problema de política pública. Raposo (2013) resaltó la relevancia del rol del estado y de la discusión de políticas públicas en torno al transporte de cargas por ferrocarril para impulsar el desarrollo económico y productivo del país.

En quinto lugar, a partir de este diagnóstico se proponen medidas de corto y mediano plazo para mejorar el servicio a partir de los cambios implementados en materia ferroviaria, portuaria y fluvial para incrementar los volúmenes transportados por estos modos.

El cambio de una matriz con pronunciada participación del modo automotor hacia una matriz más equilibrada requiere tener presente algunos desafíos. Primero, Barbero (2007) afirma que en los últimos años las firmas han modificado su operatoria en busca de reducir los tiempos de transporte y exigiendo una operatoria just-in-time, que coordine las actividades de carga-trasporte-trasbordo-descarga, desde la producción hasta los puertos. En otras palabras, en la actualidad no se busca una reducción aislada de costos de transporte sino una optimización de la logística.

El segundo desafío que se enfrenta es, no sólo la obtención de los recursos necesarios para inversiones, sino también en romper la costumbre del uso intensivo del transporte automotor. Campos, De Gus y Nombela (2003:2) destacan que para los usuarios es muy difícil cambiar el modo de transporte, dado el elevado peso de la especialización:

Las diferencias existentes entre modos de transporte son tan relevantes en la práctica que los operadores de las distintas modalidades suelen estar especializados en su parcela específica, de tal manera que, a menos que existan relaciones de complementariedad o sustituibilidad en los mercados específicos en los que operan, quienes se dedican al transporte aéreo suelen contemplar el transporte marítimo con el mismo grado de interés con el que examinan el funcionamiento de la industria farmacéutica.

Pero superados los desafíos vendrían los beneficios de una matriz de transporte balanceada hacia el modo ferroviario y fluvial. Canitrot y García (2012) analizan en detalle la vinculación entre logística y competitividad de la Argentina.

El trabajo cobra relevancia también por la coyuntura actual del país, donde Barbero (2014) estima que entre 2014 y 2003 los costos logísticos internos subieron $40 \%$ en pesos constantes. Por el lado de los ingresos, el contexto no es tan dinámico. La estabilización de los precios internacionales de los granos fue compensada con el establecimiento de un tipo de cambio real de equilibrio, acompañado de la restitución derechos de exportación para los granos. Sin embargo, si bien en el 2018 se ha observado una corrección del tipo de cambio que posiciona al país en mejores condiciones para exportar, una importante sequía ha retraído los volúmenes de producción.

Todas las expectativas están ahora puestas en la temporada 2018/2019 que, según estimaciones de la Bolsa de Comercio de Buenos Aires, alcanzará el récord de 126 millones de toneladas alcanzado en la temporada 2016/2017.

Transversalmente, este trabajo hace su aporte en términos de planificación y ordenamiento territorial, en tanto se proponen alternativas que reducen los tráficos en las rutas y descongestionan nodos que hoy están en una situación crítica. A modo de ejemplo, Barbero y Castro (2013) destacan que el nivel de presión sobre los corredores viales cercanos a los puertos de Buenos Aires y Paraná Inferior es crítico. 


\section{Producción de granos}

Como se mencionó en la introducción, los principales granos que produce nuestro país son Soja, Maíz, Trigo y Girasol y, por lo tanto, este trabajo se centrará en estos cuatro productos y sus derivados.

La producción en Argentina durante las últimas dos décadas de los principales granos registra un incremento del orden del $211 \%$, pasando desde poco más de 40 millones de toneladas en la temporada 1994/1995 a los 126 millones de toneladas que se registraron en la campaña 2016/2017, tal como lo muestra la Figura 1.

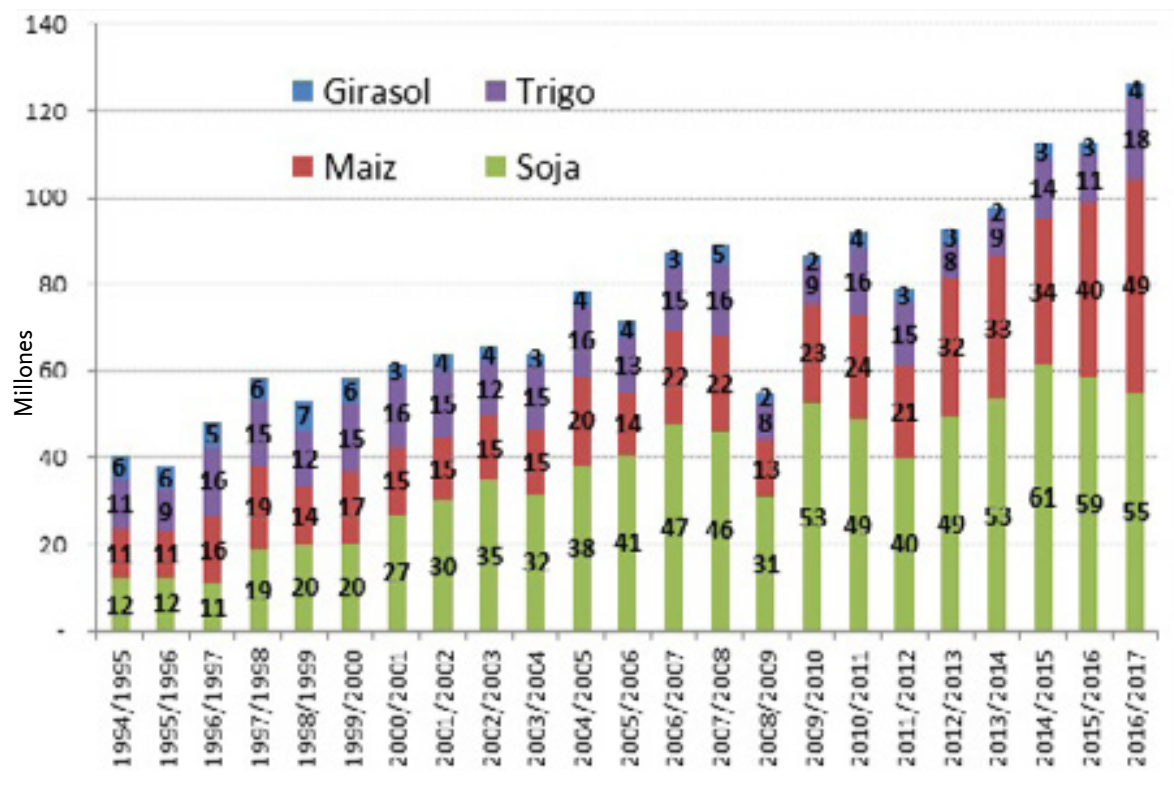

Figura 1. Evolución de la Producción de los Principales Granos 1994-2017 (en millones de toneladas). Fuente: Secretaría de Agroindustria. Consultado en marzo de 2019.

En términos porcentuales, la producción de estos granos creció a una tasa promedio geométrica de 5,3\% durante los 22 años. Este salto productivo se explica con la introducción y afianzamiento de nuevas tecnologías de producción a mediados de los años noventa. Este cambio se completa luego con un notable incremento de la demanda global de productos agropecuarios que obedece a dos factores: por un lado, la mayor demanda de proteínas por parte de los países emergentes, en especial China, y por otro el crecimiento en el uso de biocombustibles.

El tratamiento impositivo dado a partir de los derechos de exportación ha tenido varios vaivenes. Según Calzada y Rossi (2015) para el año 2015, a la soja se le aplicaba una retención del $35 \%$, al girasol $32 \%$, al trigo $23 \%$ y al maíz 20\%. Sin embargo, a finales de ese año, el Gobierno Nacional eliminó lo derechos de exportación a los granos, con excepción de la soja, tal como lo estableció el Decreto 133/2015 del Ministerio de Agroindustria. A su vez, luego de reducir de $35 \%$ a $30 \%$ los derechos de exportación de la soja, el Decreto 1343/2016 estableció una reducción mensual adicional de $0,5 \%$ desde enero del 2018 hasta diciembre de 2019, donde la alícuota quedaría estabilizada en el $18 \%$. Estas modificaciones estimularon la producción de maíz y trigo en detrimento de la soja, tal como se observa en la Figura 1.

Sin embargo, tres años después en 2018, el Decreto 793/2018 restituyó los derechos de exportación aumentando la alícuota en un 12\% (con máximo en cuatro pesos por dólar) para todos los productos, ya sean de origen agropecuario o industrial, como 
así también los servicios. Así, maíz, trigo y girasol quedaron alcanzados al 12\% y la soja al 30\%. Este cambio impositivo se dio en el medio de un deslizamiento del tipo de cambio nominal, que llevó la cotización del dólar al entorno de $\$ 40$. Lo anterior pone de manifiesto que no ha existido una política gubernamental clara orientada a impulsar la producción, procesamiento y exportación agrícola.

Por otra parte, si se realiza un análisis de la situación en particular de las provincias del NOA (Jujuy, Catamarca, Tucumán, Salta y Santiago del Estero) y NEA, (Chaco, Formosa, Misiones y Corrientes) se observa que en los últimos 20 años mostraron un mayor dinamismo, pues el crecimiento de la producción agrícola fue notablemente superior al resto del país. Tal como lo evidencia la Figura 2, mientras que las provincias seleccionadas multiplicaron su producción de estos cuatro granos casi 9 veces, el resto del país lo hizo en torno a 3 veces. Las provincias de NOA y NEA crecieron a una tasa promedio geométrico del $10,4 \%$ mientras que el resto del país lo hizo al $4,8 \%$ anual.

En consecuencia, la participación de estas regiones en la producción de granos del país pasó del 5\% al 13\%. Dentro de la región del NOA se destaca la provincia de Santiago del Estero, que paso de una producción de 414 mil toneladas en 1994/1995 a 8,2 millones en 2016/2017 con una tasa de crecimiento promedio anual del 14,6\%. En el NEA, se destaca Chaco que pasó de 447 mil toneladas a 4,2 millones, con una tasa de crecimiento anual promedio de 10,8\%. La contribución de las provincias de Jujuy, Catamarca, Formosa, Misiones y Corrientes es pequeña.

Las áreas no centrales están creciendo a tasas superiores que las centrales, lo que significa que la producción proveniente de estas regiones representa una porción mayor del total y exige ofrecer un abanico más amplio de alternativas logísticas.

La Figura 2 proporciona información adicional. No sólo el crecimiento agrícola de las provincias del NOA y NEA ha sido más veloz que el crecimiento evidenciado en el resto del país, sino que además ha sido significativamente más volátil y sinuoso.

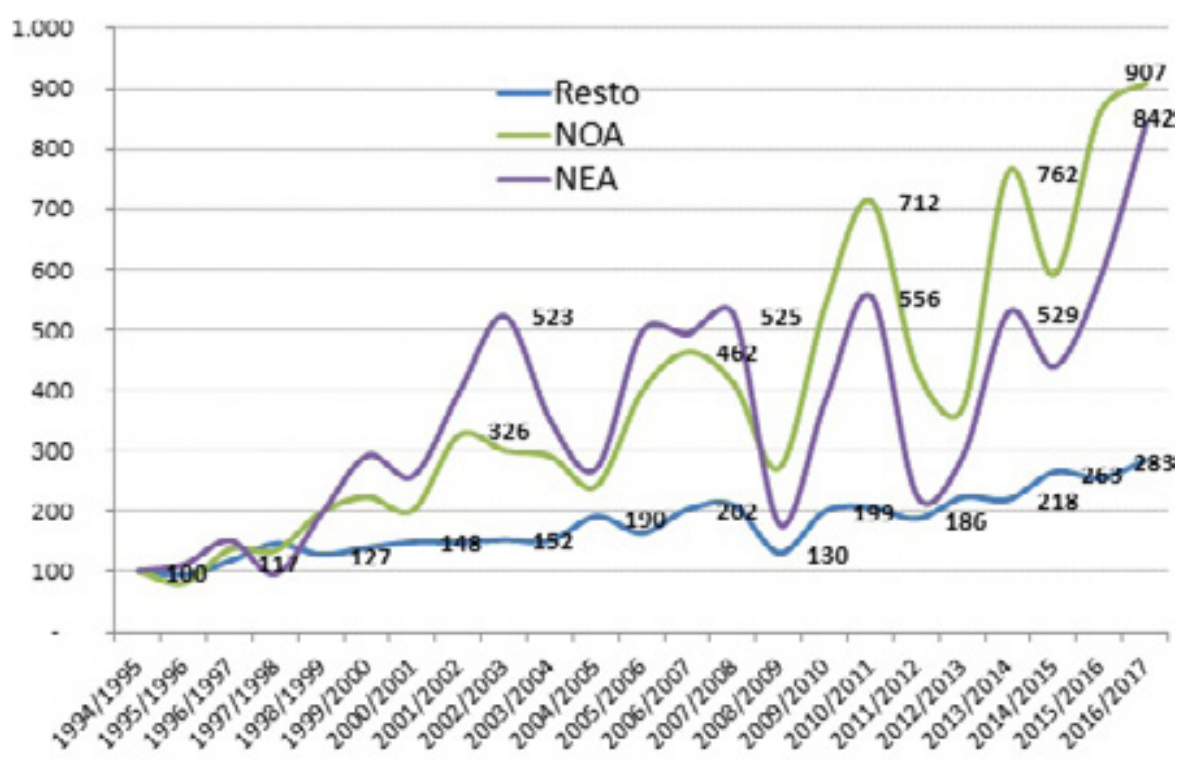

Figura 2. Evolución de la Producción de los Principales Granos NOA y NEA versus Resto del País. 1994/1995 =100. Fuente: Secretaría de Agroindustria. Consultado en marzo de 2019. 
Dos factores afectan a las regiones del NEA y NOA respecto de la producción del área central. En primer lugar, mayores distancias hasta las terminales portuarias, que elevan los costos logísticos. En segundo término, condiciones naturales y climáticas menos favorables que se materializan en rindes inferiores. Para citar algunos ejemplos, mientras que el promedio histórico del rinde por hectárea cosechada de maíz es mayor a 5 toneladas en Buenos Aires, Santa Fe y Córdoba; es de 3,4 en Chaco; 4,1 en Salta y 4,8 en Santiago del Estero. Para la soja, las provincias del centro tienen un rinde por ha. cosechada superior a las 2,5 toneladas por hectárea, mientras que Chaco tiene 1,9 y tanto Salta como Santiago del Estero 2,25.

En este sentido, ofrecer una mejor infraestructura logística que reduzca los costos podría acercar certezas respecto de la rentabilidad del negocio, reduciendo su volatilidad y favoreciendo la inversión de largo plazo.

En resumen, a pesar de los vaivenes macroeconómicos del país y de su correlato en un arancelamiento del comercio exterior de granos que no otorga certezas, la producción agrícola muestra un comportamiento dinámico. Si bien este crecimiento se evidencia en todas las provincias productoras, se destacan la incorporación de provincias del NEA y NOA, con énfasis en Santiago del Estero y Chaco. La incorporación de tierras que ofrecen menores rindes y más alejadas de las terminales portuarias del corredor Buenos Aires - Rosario, le otorga especial relevancia a la discusión respecto a la matriz de transporte utilizada en el despacho de granos y sus derivados.

\section{Transporte de los granos}

López (2012) estima que en la matriz del transporte de los granos y subproductos el camión transporta el $85 \%$ de la carga, el ferrocarril 13\%, y por la hidrovía el $2 \%$ restante. Muller y Benassi (2015) evaluaron una matriz de derivación de carga desde el transporte automotor al ferrocarril. Concluyeron que aquellas cargas generales superiores a 120.000 toneladas y a más de $500 \mathrm{~km}$ del destino, pueden cambiar de modo en un $80 \%$ de la carga. Si la carga se ubica entre las 80.000 y las 120.000 toneladas el porcentaje de derivación se reduce al $67 \%$. De esto modo, la producción agrícola descripta en la sección anterior es una carga objetivo susceptible de ser transferida de un modo a otro, por sus volúmenes y por su distancia al puerto.

La Figura 3 refleja la evolución del transporte de carga por ferrocarril desde el año 1995 hasta la fecha, considerando a los cuatro principales productos de origen agrícola y sus derivados. A diferencia de la producción de granos, los últimos años no son los record máximos en volúmenes. El transporte de granos por ferrocarriles tuvo mejor año en el 2011 cuando transportó poco menos de 13 millones de toneladas. Aproximadamente 400 mil toneladas más que los máximos anteriores de 2007 y 2010. 


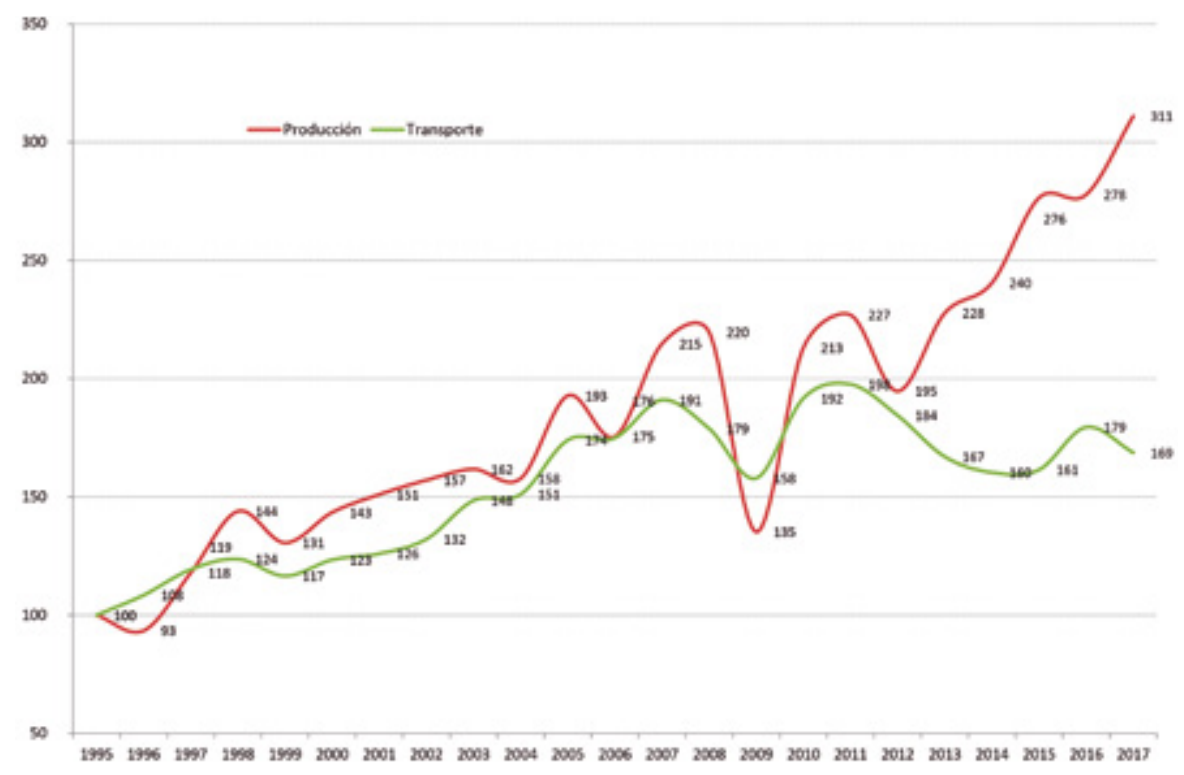

Figura 3. Evolución de la Carga de los Principales Granos 1995-2017 (en millones de toneladas). Fuente: Comisión Nacional de Regulación del Transporte. Consultado en septiembre de 2018.

Dentro del transporte por ferrocarril, la carga total muestra un decrecimiento desde los años 2005/2006, pasando de aproximadamente 24 millones de toneladas a 19 millones transportadas en 2016/2017. En el caso de los granos, el decrecimiento se da a partir del 2011 cuando se transportaron por ferrocarril aproximadamente 13 millones de toneladas y ese valor descendió a 11 millones para el 2017.

En la comparación punta a punta, el transporte de granos por ferrocarril creció a una tasa promedio geométrico de $2,4 \%$, menos de la mitad que el crecimiento en su producción. Así el ferrocarril fue perdiendo participación en el transporte total de granos, a pesar de que el mayor dinamismo en la producción se evidenció a más de $500 \mathrm{~km}$ de sus destinos para exportación y/o consumo.

Pero el desempeño no es uniforme entre todos los operadores, tal como se observa en el Cuadro 1.

Cuadro 1. Toneladas de Granos y Derivados Transportadas en Ferrocarril 1995 y 2017. Fuente: Comisión Nacional de Regulación del Transporte. Consultado en septiembre de 2018.

\begin{tabular}{|l|c|c|c|}
\hline & $\mathbf{1 9 9 5}$ & $\mathbf{2 0 1 7}$ & \% del total \\
\hline NCA SA & 2.478 .576 & 6.146 .013 & $56 \%$ \\
\hline FEPSA & 2.671 .450 & 3.296 .450 & $30 \%$ \\
\hline San Martín & 744.528 & 786.813 & $7 \%$ \\
\hline Belgrano & 251.395 & 668.336 & $6 \%$ \\
\hline Ferrosur Roca & 195.765 & 109.494 & $1 \%$ \\
\hline Urquiza & 182.143 & - & $0 \%$ \\
\hline
\end{tabular}

Nuevo Central Argentino SA (NCA), el principal operador, tuvo un crecimiento promedio geométrico de 4,2\% pasando de 2,5 millones de toneladas en 1995 a 6,1 en 2017. En el 2017 NCA transportó el 56\% de los granos transportados por ferrocarril. 
Ferroexpreso Pampeano SA (FEPSA), el segundo operador de granos con el 30\% de la carga transportada en 2017, tuvo un crecimiento promedio geométrico de $1 \%$. En 1995 transportó 2,7 millones de toneladas y en 2017 3,3 millones.

La Línea Belgrano operada por la empresa estatal Belgrano Cargas, transportó apenas 670 mil toneladas de granos (6\%) en 2017 y creció a una tasa promedio geométrica de 4,5\%. La Línea San Martín con 780 mil toneladas (7\%) transportadas creció al 0,21\%. Ferrosur Roca SA transporto 110 mil toneladas en el 2017, lo que significa un decrecimiento promedio del -2,6\%. La Línea Urquiza dejó de transportar granos en el 2015. Debido a la baja participación de estas líneas en el total de la carga de granos no serán tenidas en cuenta a lo largo de este trabajo, dado que no resulta relevante realizar una descripción del recorrido que, individualmente, realiza menos del 10\% de la carga. Esta situación no implica que una estrategia que busque optimizar la logística argentina considere la utilización de la infraestructura que estas líneas tienen disponibles.

Si bien los dos principales operadores de granos incrementaron su carga en los últimos 20 años, este incremento fue inferior al crecimiento de la producción agrícola. Además de esa brecha, los operadores más pequeños han tenido reducción de los volúmenes de carga. Por último, también hay que considerar que respecto del 2011 todos los operadores han reducido el volumen total de carga, aun cuando la producción agrícola siguió en ascenso.

La evolución del transporte por ferrocarril de los granos seleccionados y sus derivados vis a vis la evaluación de la producción se muestra en la Figura 4. Se observa con claridad como a partir del 2011 este modo de transporte ha perdido relevancia.

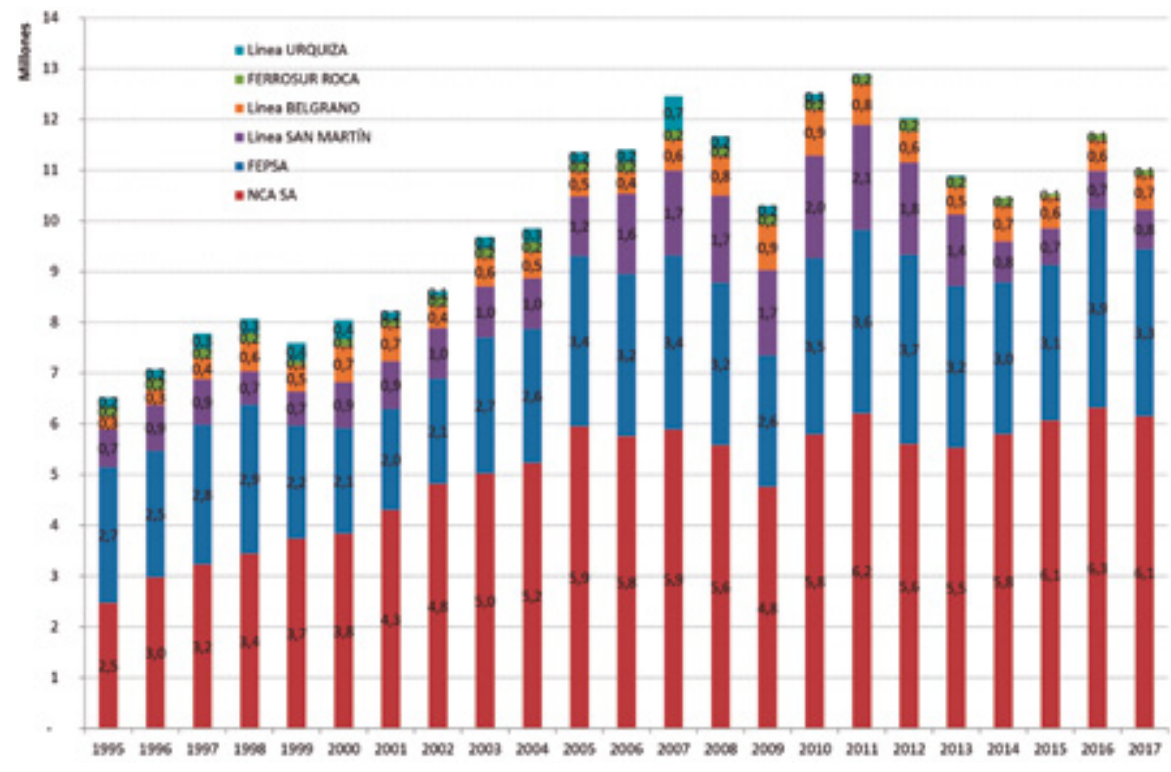

Figura 4. Evolución de la Producción y Carga de los Principales Granos 1995=100. Fuente: Comisión Nacional de Regulación del Transporte y Secretaría de Agroindustria. Consultado en septiembre de 2018.

La figura muestra que hasta el año 2009, la evolución de la carga y la producción tuvieron un comportamiento similar. Justo ese año la evolución acumulada de carga superó a la evolución de la producción, esta última afectada seriamente por la sequía de esa temporada. A partir de allí, la producción no solo recuperó los volúmenes previos sino también que se mantuvo en crecimiento, mientras que a partir del 2011 la carga tiene un retroceso.

Uno de los aportes de este trabajo, tal como se mencionó en el resumen, es la construcción de un Índice de Penetración del Ferrocarril en el transporte de granos y derivados. 
El indicador es definido como el cociente entre las toneladas de producción de granos y la cantidad de toneladas de granos y subproductos transportadas por ferrocarril de carga, ambas magnitudes en base 1995 $=100$.

La necesidad de este indicador radica en la diversidad de unidades de medida utilizadas para presentar la información. La producción de granos esta expresada en toneladas de cada variedad de grano. Pero la carga esta expresada, a veces, sin discriminar que granos son, otras veces de aceite (de soja, de girasol, de maíz o mezcla), otra veces de pellet y/o harina. Estas diferencias de exposición, sumadas a la posibilidad de acumular stocks en silo bolsas, impiden reconstruir una trazabilidad exacta para los granos. Los resultados para este índice, se exponen en la Figura 5.

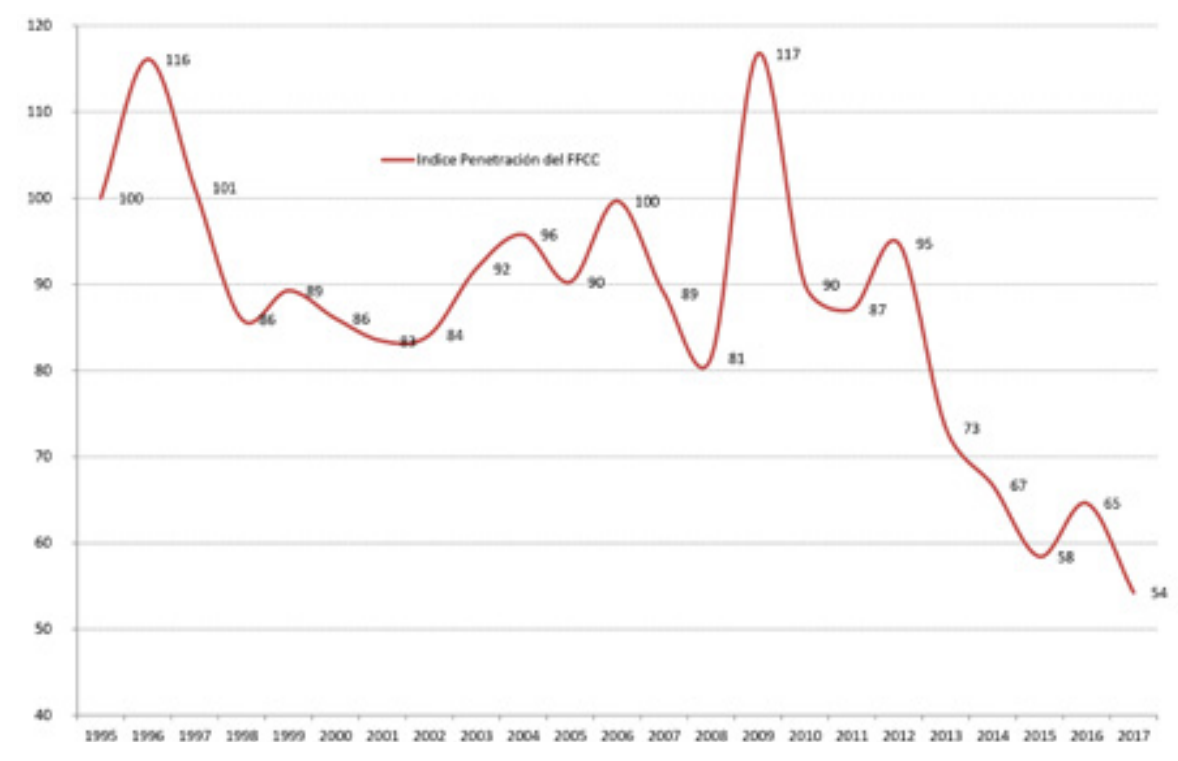

Figura 5. Evolución del Índice de Penetración del Ferrocarril en la Carga de los Principales Granos 1995=100. Fuente: Elaboración Propia en Base a Comisión Nacional de Regulación del Transporte y Secretaría de Agroindustria y Secretaría de Agroindustria.

El Índice de Penetración del Ferrocarril en la carga de granos permite sacar algunas conclusiones respecto de su evolución reciente. Los volúmenes cargados de granos y derivados han tenido un crecimiento lento comparado con el crecimiento de la producción agrícola. Esta brecha es aún mayor si se la comparase con las regiones de NEA y NOA.

Esta situación implica que la carga en ferrocarril va perdiendo peso relativo, que sólo se recuperan en los años en los cuáles la producción se ve reducida por una sequía. Vale una mención respecto de la coyuntura. Si bien la capacidad de carga se reduce a partir del 2011, la sequía de la temporada 2017/2018 permite suponer una recomposición del índice de Penetración para el 2018.

En la sección anterior se ha mostrado con datos estadísticos que la producción agrícola de la Argentina esta en expansión, y que esa expansión es más veloz en zonas alejadas de las terminales portuarias.

En esta sección, se contextualizada la carga de granos y derivados en el ferrocarril. El crecimiento de este modo de transporte fue más bajo que el crecimiento de la producción de granos, lo que significa una pérdida de participación. Esta evolución en el transporte por ferrocarril no es homogénea entre los distintos operadores, lo que significa un impacto territorial dispar. 
A este punto de la exposición, es evidente la complejidad de la situación actual. La producción de granos crece sostenidamente y con mayor velocidad en zonas alejadas de los puertos. Esta situación hubiese permitido suponer un uso más intensivo de modos de transporte como el ferrocarril (o, de ser posible, el fluvial) más eficientes que el automotor para largas distancias.

Sin embargo, los datos muestran que el crecimiento en el transporte de carga por ferrocarril es más lento que la producción a agrícola, y, sobre todo, significativamente más lento que el crecimiento de las zonas alejadas del corredor Buenos Aires - Rosario. Peor aún, el transporte de granos por ferrocarril está en retroceso desde el año 2011.

En la próxima sección se mostrarán los datos de producción de granos, movimientos a través del ferrocarril de carga en las tres regiones geográfica analizadas: centro, NEA y NOA.

\section{Flujo de granos y derivados}

Cómo herramienta adicional para profundizar el análisis de la dinámica del transporte de granos y derivados por ferrocarril se trabajó en interpretar las matrices de Origen y Destino (OD) para los granos y sus derivados (aceites, harinas y pellets).

\section{Buenos Aires y Centro}

La red FEPSA tiene dos destinos, Buenos Aires y Rosario. Se muestran los datos para 24 estaciones de origen que representan más del $75 \%$ de la carga de granos, ninguna de las estaciones omitidas reunía el $1 \%$.

La Figura 6 se elaboró a partir de la matriz OD, donde las circunferencias rojas indican los puntos de origen y las celestes los puntos de destinos. El tamaño de la circunferencia guarda relación con los volúmenes cargados o descargados.

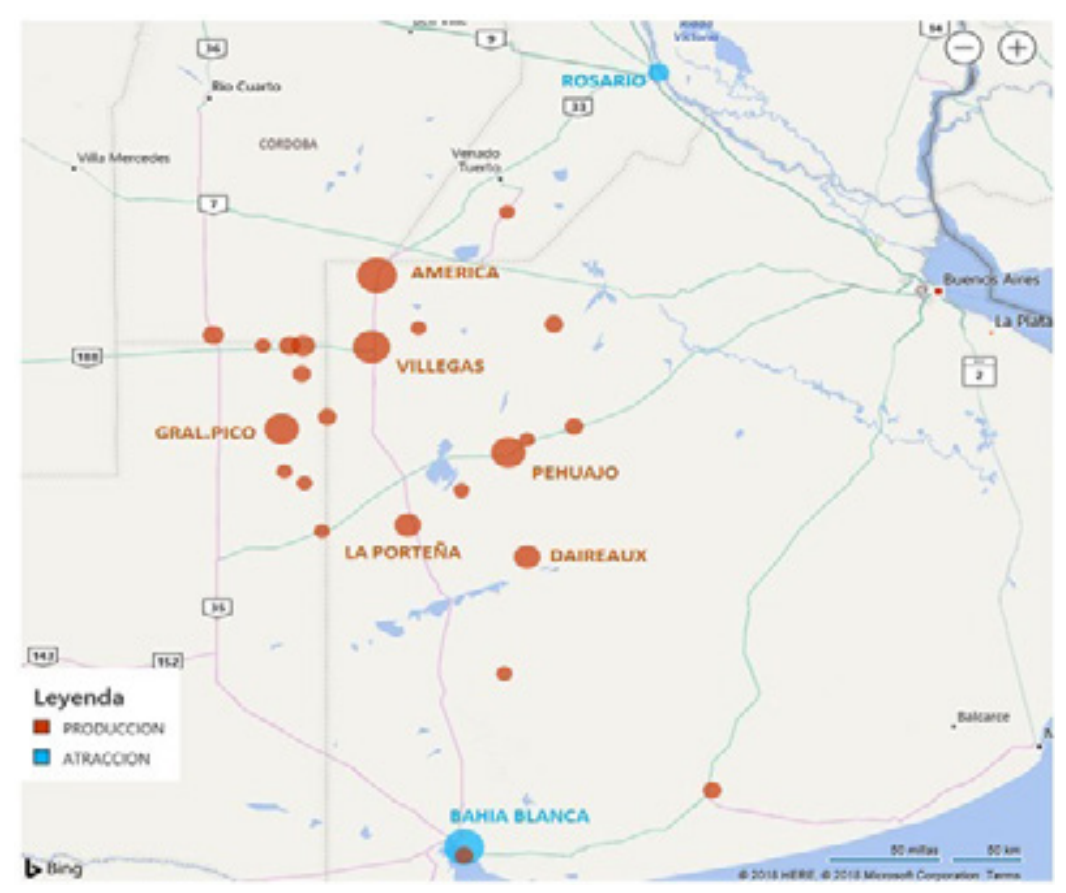

Figura 6. Representación Geográfica de la Matriz de Origen (Producción) y Destino (Atracción) de FEPSA para los principales granos y derivados. Fuente: Elaboración Propia. 
La red que fue concesionada a Ferro Expreso Pampeano (FEPSA) es extensa, con más de $5.000 \mathrm{~km}$ de vías de las cuales, y casi 3.000 que se encuentran operativas. Se encuentra principalmente localizada dentro de Buenos Aires, la provincia con mayor producción agrícola del país (casi 40 millones de toneladas y el 31\% de la producción total en el 2017). La traza de sus vías se ubica en el torno de la recta que une la ciudad de Bahía Blanca con Rosario. Por lo tanto, las vías recorren la provincia por el lado oeste, facilitando la carga del este de La Pampa y de San Luis más el sur de Córdoba y Santa Fe.

No se consideran los volúmenes de Ferrosur Roca, que transportó apenas 100 mil toneladas en 2017, aproximadamente la mitad de los transportado 20 años atrás. Ferrosur Roca está integrado verticalmente con la cementera Loma Negra y se dedica a transportar principalmente materiales para la construcción. Además, al tener la traza de sus vías en el este de la provincia de Buenos Aires, la distancia a los puertos (principalmente Bahía Blanca y Quequén es todavía menor).

Sin embargo la participación de lo transportado por la red FEPSA es muy baja respecto de la producción total. Tal como se sostuvo la sección correspondiente, para el año 2017 transportó 3,3 millones de toneladas y muestra un crecimiento del 1\% en los últimos años.

La distancia promedio de cada estación a su destino no supera los $400 \mathrm{~km}$, reduciendo aprovechamiento de las ventajas que el modo ferroviario ofrece en distancias más largas. Si bien los volúmenes son muy grandes, el camión tiene la ventaja logística de reducir la carga-descarga a una operación. Al ser necesario el flete corto desde la tranquera hasta la estación del tren, las operaciones de carga-descarga se incrementan al menos en dos intervenciones.

En palabras más sencillas, por un recorrido de $400 \mathrm{~km}$ una vez que ya se subió la mercadería al camión es más práctico enviarla a destino, que descargarla en una estación para volver a cargarla en el tren.

A esta desventaja hay que sumarle el cambio en el modelo de negocio que ha sufrido la actividad agrícola a partir de la incorporación de silos bolsas. Con anterioridad era común que el acopio de los granos se realice en un silo cercano a la estación, lo que facilitaba su posterior carga al ferrocarril. Con los silos bolsas, el copio se hace en cada campo dándole mayor capilaridad a la producción y dificultando el acceso del ferrocarril.

La zona de influencia de FEPSA, descripta en la Figura 7, está constituida por 55 partidos, de los cuáles 36 son de Buenos Aires, 11 de la Pampa, 5 de Santa Fe y 3 de Córdoba. En toda el área se producen más de 42 millones de toneladas de granos. Estos volúmenes permiten pensar una estrategia que compense las relativamente cortas distancias con mayor carga. De hecho, las Matrices de Orígenes y Destinos de Cargas (2017) elaboradas por el Ministerio de Transporte muestran el congestionamiento que el modo automotor está generando en los alrededores de la ciudad de Rosario. 


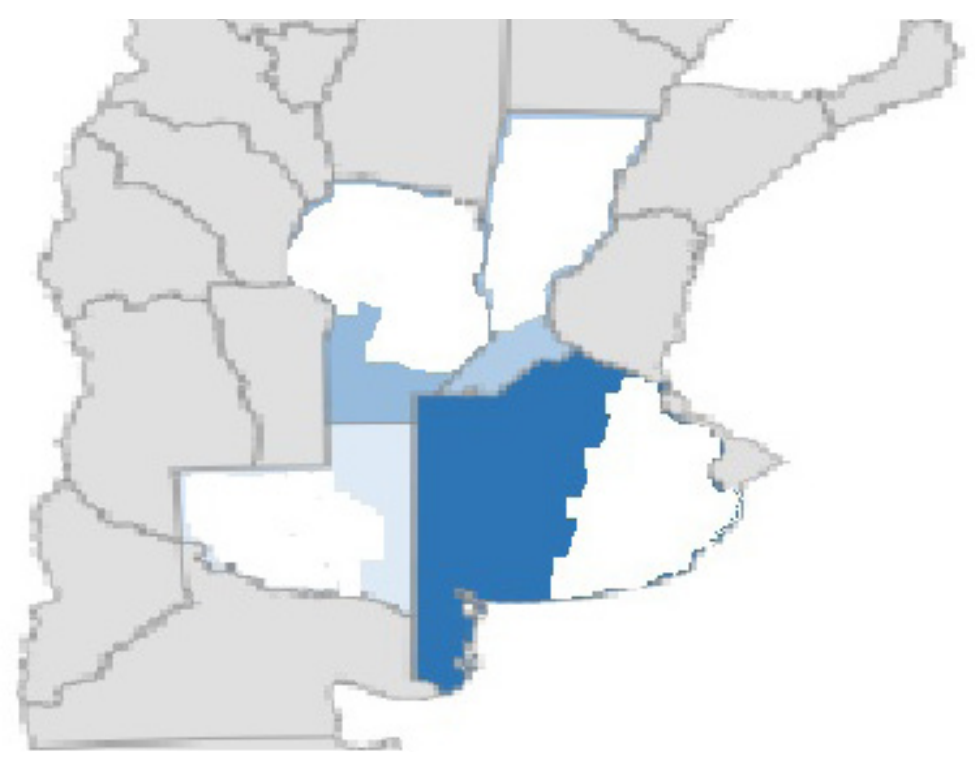

Figura 7. Producción de los Granos en la Zona de Influencia de FEPSA 2016/2017. Fuente: Elaboración propia en base a Secretaría de Agroindustria.

\section{Región NOA}

Un análisis similar se presenta para otra concesión del transporte ferroviario Nuevo Central Argentino (NCA). Esta concesión posee una red que atraviesa zonas del NOA, donde la agricultura ha crecido estos últimos años a tasas muy elevadas y cuyos granos deben recorrer largas distancias hasta el destino final.

En la Figura 8 se muestran se los puntos de origen y destino (producción y atracción) correspondientes a la Matriz OD de esta concesción.

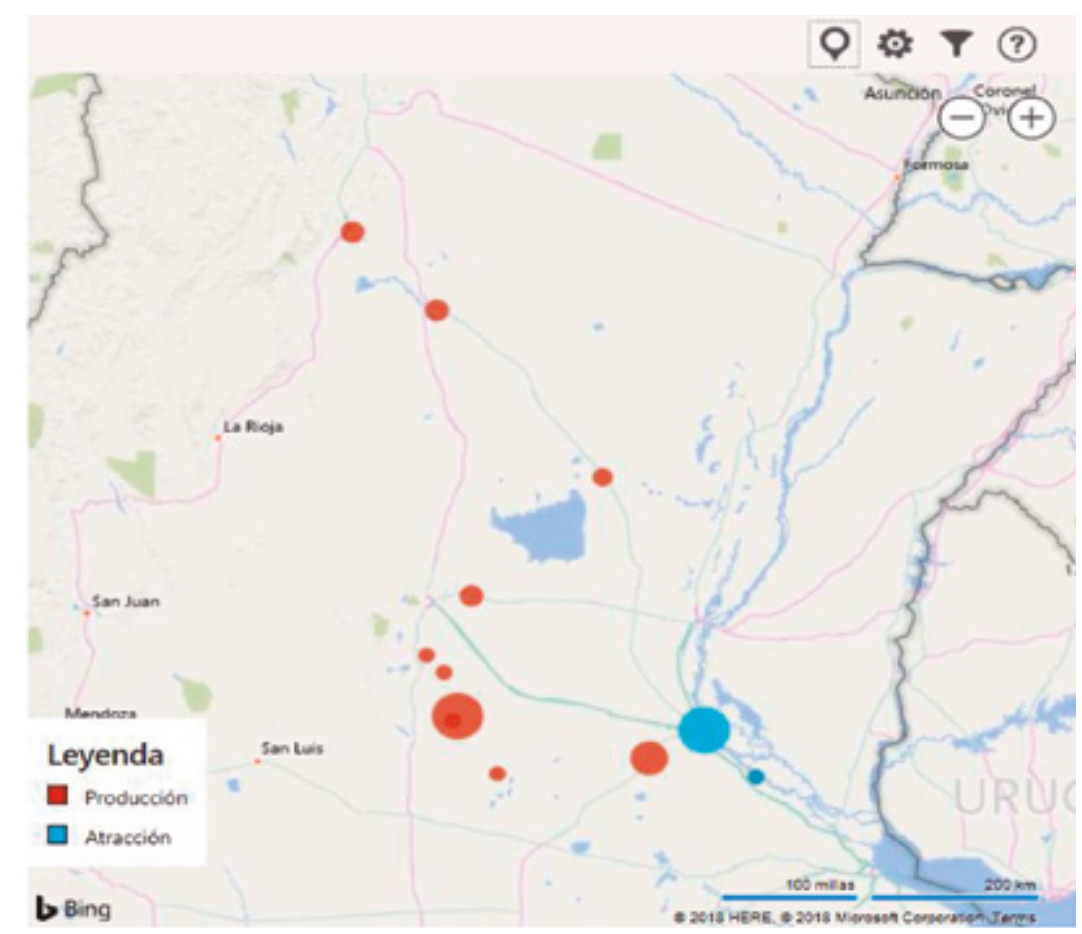

Figura 8. Representación Geográfica de la Matriz de Origen (Producción) y Destino (Atracción) de NCA para los principales Granos. Fuente: Elaboración Propia. 
La red concesionada a NCA tiene una extensión de $4.750 \mathrm{~km}$ de los cuáles 3.200 se encuentran operativos. La traza de sus vías atraviesa las provincias de Santa Fe, Córdoba, Santiago del Estero y Tucumán.

Como se mencionó anteriormente, en el año 2017 esta concesión transportó 6,1 millones de toneladas de granos y subproductos, representando el $55 \%$ de este tipo de cargas en el ferrocarril. Además, mostró una tasa de crecimiento geométrico de 4,2\% en los últimos años.

Si bien esta tasa de crecimiento es la más alta dentro de los operadores, es inferior a la tasa de crecimiento promedio de la producción agrícola. Y, como se observa, sensiblemente inferior a la tasa de expansión del agro en la región NOA.

NCA tiene la particularidad de estar integrada verticalmente con la empresa Aceitera General Deheza (Grupo AGD). Por lo cual, los principales puntos de origen y destino se corresponden con la dinámica económica de ese grupo. AGD tiene sus principales platas procesadoras de granos en las localidades Gral. Deheza (Córdoba) y Chabas (Santa $\mathrm{Fe}$ ) y también tiene un centro de acopio de granos en Beltran (Santiago del Estero). Adicionalmente va cargando granos en distintas estaciones de la región centro y NOA, por ejemplo, en Cevil Pozo (Tucumán) carga una parte de la producción de esa provincia.

El destino final es la ciudad de Rosario, donde AGD tiene su planta industrial más importante, denominada terminal 6. Un destino secundario es la ciudad de Ramallo donde está localizada una planta de procesamiento y exportación de Bunge.

La distancia promedio entre las principales estaciones supera los $450 \mathrm{~km}$ con picos en el ramal norte (Tucumán): 1050 km entre Cevil Pozo y Ramallo; 950 km entre Cevil Pozo y Rosario; 850 km entre Beltrán y Ramallo y 750 km entre Beltrán y Rosario.

Si se observa el cordón oeste (Córdoba) de esta concesión, el ferrocarril podría tener una importante capacidad competitiva para canalizar las producciones que se encuentran al oeste (por ejemplo, la Provincia de San Luis). En los últimos años se han realizado inversiones en mejoras, iniciando el trabajo de renovación de las vías del tramo Córdoba - Rosario, empezando por el tramo Rosario - Tortugas, lo que habilita un mejor aprovechamiento de la red.

Federman, Mangiameli y Marcó (2018) han analizado algunas particularidades de la integración de ambas empresas en otro trabajo. Si bien no se desarrollan de nuevo en éste, algunas consideraciones adicionales merecen ser realizadas. En primer lugar, para el caso particular de los granos, la asociación de un operador ferroviario de cargas con una empresa de producción y molienda de granos, sirvió para darle impulso a la carga, impulso que no se evidenció en otras concesiones. En segundo lugar, la presencia de grandes volúmenes y distancias favorecen el uso de ferrocarril.

En la figura 9 se muestra la zona de influencia del ramal norte de la concesión de NCA. 


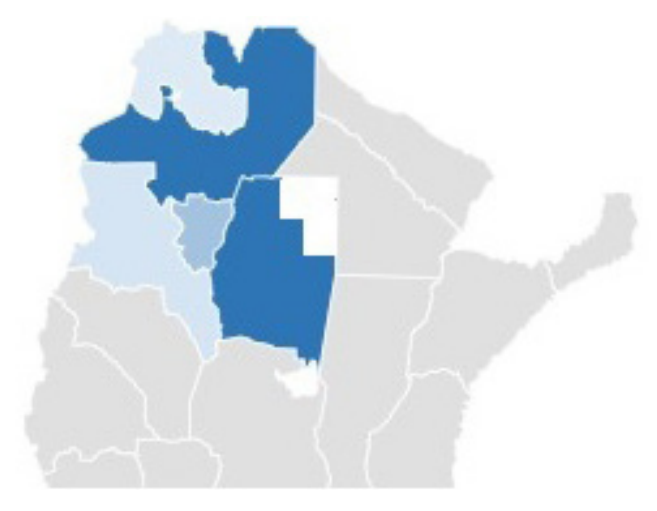

Figura 9. Producción de los Granos en la Zona de Influencia de NCA Ramal Norte 2016/2017. Fuente: Elaboración propia en base a Secretaría de Agroindustria.

Para el cálculo de los volúmenes máximos que se podrían transportar por este ramal se tomó la producción total de los principales granos en las provincias de Tucumán, Salta, Jujuy y Catamarca. De la provincia de Santiago del Estero se excluye la producción de tres partidos del noreste, que serán considerados en la región NEA. Así hay registro de 60 partidos activos agrícolamente en la temporada 2016/2017.

Las provincias del NOA seleccionadas aportaron 8,9 millones de toneladas en esa temporada. Suponiendo una distancia promedio de $900 \mathrm{~km}$ al puerto de Rosario, equivalen a más de 8.000 millones de toneladas-kilometro.

Estos volúmenes son atractivos a la hora de plantear políticas orientadas a mejorar la logística del país. Resulta económicamente razonable incurrir en un flete corto y una operación de carga-descarga adicional si la mercadería será transportada por ferrocarril una distancia de $900 \mathrm{~km}$. Calzada y Sese (2017) de la Bolsa de Comercio de Rosario estiman que el ahorro en flete para granos de esta región llega al 19\% del valor neto del trigo, $17 \%$ del maíz y $9 \%$ de la soja.

Para el cálculo de los volúmenes máximos que se podrían transportar por el ramal centro se tomó la producción de los principales granos en las provincias de San Luis, Córdoba y Santa Fe pero excluyendo aquellos partidos que ya fueron considerados para FEPSA o aquellos lejanos a la vía (un total de 38 partidos). Estas provincias del centro seleccionadas aportaron 38,7 millones de toneladas en esa temporada. Vale destacar que en estos casos la distancia promedio se reduce sensiblemente.

\section{Región NEA}

La red ferroviaria que atraviesa la región del NEA es la Línea Urquiza. Como se mencionó anteriormente esta línea dejó de transportar granos en el año 2015, dedicándose principalmente al transporte de minerales y maderas.

Esta línea se encuentra en peores condiciones de operación que el resto, y su puesta a punto requeriría de fuertes inversiones. Según datos de la CNRT, es la red con menor cantidad de km operativos (1.486), menor cantidad de locomotoras en funcionamiento (32) y de vagones (1.521). Además, es la red con menor cantidad de pasos a nivel (1019), de los cuáles del 94\% no tienen barreras. En el año 2017 transportó menos de 200 mil toneladas.

Por lo tanto, resulta razonable centralizar la producción de granos en el puerto de Barranqueras (Chaco) y, llegado el caso de Corrientes, realizando las obras necesarias para que los barcos que allí carguen puedan operar a través de la Hidrovía Paraná 
- Paraguay. Para esto es necesario que las obras no sólo garanticen la correcta navegabilidad, sino que también los puertos dispongan de la burocracia administrativa necesaria, como una delegación de Aduana, del Servicio Nacional de Sanidad y Calidad Alimentaria (SENASA) etc. para que los productos se embarquen directamente allí. Si el Estado da una señal de largo plazo en este sentido, los privados deberían comprometer la construcción de una planta de almacenamiento y moliendo en las cercanías del puerto.

El desarrollo de este puerto se empalma con un posible resurgimiento de la Línea Belgrano, cuya traza (Ramal Norte) atraviesa Salta, el norte de Santiago del Estero y desemboca en esta ciudad chaqueña. Esta línea esta recibiendo importantes inversiones por parte del Estado y los volúmenes muestran un buen desempeño. En los primeros 4 meses del 2019, se transportaron 339 mil toneladas de granos, $40 \%$ más que las transportadas en los primeros 4 meses del 2018.

En esta región se considera la producción de tres paridos del noreste Santiago del Estero (que fueron excluidos de la región NOA), tres partidos del norte de Santa Fe y la totalidad de la provincia de Chaco. Las provincias de Formosa, Corrientes y Misiones tienen una contribución muy pequeña a la producción de estos granos.

La Figura 10 muestra la distribución en esta región, que totaliza 9,7 millones de toneladas correspondientes a la temporada 2016/2017.

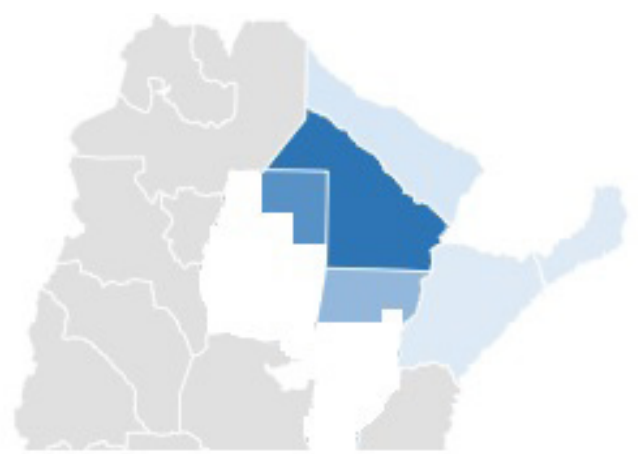

Figura 10. Producción en la Zona de Influencia del Puerto de Barranqueras - Corrientes. Fuente: Elaboración propia en base a Secretaría de Agroindustria.

Las concesiones no pudieron capturar una porción importante del significativo crecimiento de la producción agrícola del país, en especial del rápido crecimiento de las zonas más alejadas y que más necesitan de este modo de transporte.

La única excepción viene de la mano de la red concesionada a NCA, cuya integración vertical con una empresa agroindustrial le permitió tener un desempeño alineado con la dinámica del agro.

A su vez, la expansión agropecuaria del NEA, con epicentro en la provincia del Chaco demanda de un Estado más activo en términos de infraestructura exportadora. La localización de un puerto permitiría limitar el transporte terrestre (ya sea ferroviario o automotor) y descomprimir el congestionamiento de la zona portuaria de Rosario.

Por lo tanto, es necesario generar el marco y las políticas de largo plazo adecuadas con el fin de lograr mayor participación de los modos ferroviario y fluvial en el transporte de los granos, cómo forma de apuntalar la competitividad y el crecimiento de las regiones alejadas del centro agrícola del país. 


\section{Regulación y marco normativo}

El primer aspecto por destacar respecto de la regulación es el proceso concesión a privados de la década del 90. Este proceso se inició con la Restructuración Ferroviaria establecida por el Decreto 666/89 y se previó que el transporte de carga priorizara el modelo de concesiones a empresa.

Raposo (2013) detalla al modelo concesionario de la siguiente manera. Las concesiones establecían un canon por el uso de la red, sus instalaciones y su material rodante y un plan de inversiones para la actualización y renovación del sistema. El concesionario estaba a cargo de la explotación comercial, la operación de los trenes, la atención de las estaciones y el mantenimiento del material rodante. El Estado mantenía la propiedad y la supervisión regulatoria.

De este modo, y en lo relativo a la analizado en este trabajo, FEPSA obtuvo la línea Rosario - Bahía Blanca en 1991 y NCA SA se adjudicó la Línea Mitre en 1992. Estos cambios generaron impactos dispares por operador. NCA SA aumentó sus toneladas transportadas totales de 3,5 millones en 1994 a 6,2 en 2001 (+77\%). FEPSA se mostró una leve caída, pasando de 2,5 millones a 2,4 (-4\%) en las mismas fechas.

Una consecuencia de la concesión a privados del transporte ferroviario de cargas fue la especialización. NCA SA, que esta integrada verticalmente con AGD SA, mantuvo la proporción de granos y subproductos transportados. En 1995, 2,5 toneladas de los 3,5 totales se correspondían con estos productos (71\%) mientras que en 20014,3 de los 6,2 totales eran granos y subproductos (69\%).

En FEPSA, que se encuentra integrada verticalmente con el Grupo Techint, los granos y derivados perdieron relevancia. En 1995 se transportaron 2,7 toneladas en un total de $2,9(93 \%)$ y en 20012,0 de un total de 2,4 (83\%).

En simultaneo con la dispar evolución de la carga transportada y una especialización condicionada por la integración vertical del explotador, Sharp (2005) destaca que se incumplieron los compromisos de inversión dando lugar a un proceso de descapitalización del ferrocarril.

Estos incumplimientos en conjunto con la crisis económica del 2001 dieron lugar al inicio de un proceso de constantes renegociaciones de los contratos de concesión. Estas negociaciones se dieron en el ámbito de la Unidad de Renegociación y Análisis de Contrato de Servicios Públicos (UNIREN), una unidad creada especialmente para rediscutir los términos de las concesiones de aquellos servicios públicos que se vieron afectados por la salida abrupta de la convertibilidad.

Raposo (2013) destaca cuatro puntos salientes sobre los cuales los concesionarios se beneficiarían luego de la renegociación de los contratos: a) quedaron eximidas del pago del canon, b) se condonaban obligaciones de inversión fija y los nuevos compromisos eran proporcional a la facturación (10\%), c) el estado asumía el compromiso de mantener las vías y d) asignar los montos correspondientes a multa a inversión.

Posteriormente la Ley 26.532 del 2008 crea dos nuevas Sociedades del Estado. Administración de Infraestructuras Ferroviarias SE (ADIFSE) absorbe la administración y mantenimiento de la infraestructura, la cual queda inventariada en Ferrocarriles Argentinos. Sociedad Operadora Ferroviaria SE (SOFSE) asume la prestación de servicios, ya sea de pasajeros o carga. La sanción de esta ley coincidió con el volumen máximo transportado por ferrocarril de cargas, casi 25 millones de toneladas en 2007 y 23,6 en 2008. 
A través del Decreto 566/2013, se crea Belgrano Cargas y Logística SA (BCyL), con el objetivo de potenciar los ferrocarriles de carga en beneficio nacional y federal, asignándole a esta sociedad del Estado la administración de las tres líneas detalladas a continuación. La empresa es de capital 100\% estatal, estando sus acciones en poder de la SOF S.E., AGP S.E. y ADIF S.E., y tiene a su cargo las líneas Belgrano, San Martín, y Urquiza.

El marco normativo y regulatorio del transporte de carga tuvo una nueva modificación con la sanción de la Ley 27.132, con fecha el 15 de abril de 2015. Fue fundada en la necesidad de una política de reactivación de los ferrocarriles de pasajeros y de cargas y en la renovación y mejoramiento de la infraestructura ferroviaria.

Entre los puntos más salientes de esta ley, pueden resaltarse los siguientes.

" Se declara de interés público nacional a los ferrocarriles de pasajeros y de cargas y se establece como objetivo prioritario su reactivación (Art. 1).

» Se establecen lineamientos generales para la administración de la infraestructura por parte del Estado Nacional y se habilita la participación pública y privada en la operación de los servicios públicos ferroviarios.

»Se reasume la plena administración de la infraestructura ferroviaria y la gestión de los sistemas de control de circulación (Art. 3).

»Se determina la necesidad de promover condiciones de libre accesibilidad (open access) a la red ferroviaria para servicios de cargas. Para ello se establece la creación de un Registro de Operadores de Carga. (Art. 4).

»Se constituye Ferrocarriles Argentinos Sociedad del Estado (FASE) en la órbita del Ministerio del Interior y Transporte. (Art. 5). FASE tendrá el 100\% de las acciones de ADIFSE y SOFSE y la participación del Estado en las concesionarias de carga ( $16 \%$ de NCA, FEPSA y FERROSUR ROCA).

$\mathrm{Al}$ igual que lo sucedido en el comercio exterior de granos, el conjunto de regulaciones que el Estado ha introducido en lo relativo a la carga por ferrocarril, lejos de ser unísonas se caracterizan por pronunciados vaivenes.

Estas idas y vueltas han arrojados resultados dispares en el tiempo y por operador. Si bien con las privatizaciones se logró un aumento en los volúmenes de algunos operadores, también se dotó al sistema de una visión cortoplacista con bajos incentivos a desembolsar sumas en inversión y mantenimiento. Y generó una especialización en función de la integración vertical a nivel firma en lugar de priorizar el entorno económico y territorial de la traza de las vías.

El quiebre de la convertibilidad y la posterior renegociación de los contratos permitió que los operadores siguieran sumando cargas, hasta alcanzar el record en el año 2007. A partir de la ley del año 2008, el ferrocarril entro una fase de descenso en volumen. Los granos y sus derivados solo pudieron mantenerse al margen de esta tendencia decreciente hasta el año 2011.

Es importante recordar que, si bien la ley del 2015 establece un nuevo marco institucional para la carga en ferrocarril, todavía no se consta con datos estadísticos de suficiente extensión temporal para evaluar su impacto. Además, la ley fue sancionada pero no fue reglamentada, dificultando la obtención cualquier conclusión al respecto debido a una posible ausencia de causalidad.

Por lo expuesto, es necesario un marco normativo que apunten a resolver las principales limitaciones del transporte de carga. El eje central de la regulación tiene que pasar por otorgar certezas, tanto a los oferentes de carga como a los dadores de carga respecto de 
las regulaciones en el largo plazo. Recordando lo que fuese citado en la introducción, que es dificultoso para una firma cambiar de modo de transporte y solo evaluará cambiarse frente a un oportunidad más económica y persistente en el largo plazo.

Pero también hay que tener presente que existen otras limitaciones que no provienen de cuestiones regulatorias. A saber: a) problemas de coordinación entre productores y concesión ferroviaria, b) ineficiencias en la carga y en la descarga, c) fluctuaciones en los volúmenes producidos y d) limitaciones en los puertos.

\section{Propuestas}

En esta sección del trabajo, se postulan algunas propuestas para un mejor aprovechamiento de la infraestructura existente en conjunta con una selección de posibles obras que mejoren la operación y explotación. Estas propuestas se presentan separadas en aquellas que son plausibles de aplicarse en el corto plazo y aquellas de mediano o largo plazo.

\section{De Corto Plazo}

Para el corto plazo, se proponen medidas que permitan recuperar los volúmenes transportados en el año 2011, unas 2 millones toneladas más que las transportadas en el 2017. Se trata de propuestas que no apuntan a una expansión de la infraestructura del país, ya que en el pasado se ha transportado este volumen. Son propuestas principalmente de coordinación y que tiene como horizonte temporal de 1 a 2 años.

》Registro de demanda. Como se mencionó anteriormente, este registro fue propuesto en la Ley 27.132 pero su implementación viene demorada. Si bien varias veces fue anunciado por autoridades del Ministerio de Transporte, todavía no está en práctica ${ }^{1}$. Este trabajo sugiere la existencia de demanda insatisfecha, en tanto la producción agrícola crece más rápidamente en las regiones lejanas a los puertos. Constituir un registro de demanda le permitiría al Estado confirmar la hipótesis de este trabajo como así también conocer con una precisión aún mayor la especificidad de esta demanda. Adicionalmente este registro puede brindar información acerca de los motivos por los cuales no se presta el servicio.

La disposición de esta información habilitaría diseñar políticas que subsanen las deficiencias, en particular asegurar la accesibilidad de todos los jugadores al sistema. A lo largo de este trabajo se hizo hincapié en la necesidad de una regulación que dé certezas a los operadores y, por lo tanto, el eje de las nuevas políticas debe estar puesto en la coordinación y no en la imposición.

"Coordinación de potenciales cargadores. El Estado debe articular compromisos de mediano plazo entre las partes, que generen garantías y previsibilidad en la continuidad del servicio a lo largo del tiempo. Los demandantes de cargas deben asegurar un volumen mínimo y sostenido en el tiempo, mientras que los oferentes deben asegurar la infraestructura para brindar el servicio. A cambio, los demandantes deberán pagar la tarifa por utilización de la red que será recibida por el concesionario, la cual es inferior a los actuales costos de transporte por la vía automotor.

"Publicación de los costos marginales de utilización. Como parte de la coordinación se deben dar a conocer los costos marginales de la utilización de la infraestructura y de los servicios de transporte según distancia y tipo de producto. Para que la 
negociación entre dadores y receptores de carga sea transparente y se apoye sobre los costos de producción y la rentabilidad propia de un mercado con competencia.

"Acuerdos sectoriales. Un paso posterior de mayor alcance sería alentar y participar en acuerdos sectoriales entre las empresas de logística y los sectores productivos, que garanticen cupos de carga y tarifas competitivas

\section{De Mediano y Largo Plazo}

Con estas medidas se apunta a que el ferrocarril y el transporte fluvial ganen participación en la matriz logística del sector agroindustrial. Su horizonte es de 3 a 10 años.

Suponiendo que la producción de granos sigue creciendo al 5\% y partiendo de una producción actual de 100 millones de toneladas, en 10 años la producción podría alcanzar 162 millones de toneladas. Si se lograse que la participación del ferrocarril (y todo caso el transporte fluvial) llegasen a 30 millones de toneladas, el Índice de Penetración daría 115, un valor similar al observado en 2009.

》Infraestructura Ferroviaria: El Estado debe definir con precisión las prioridades de inversión, con un enfoque de corredores productivos con sentido estratégico. En ese sentido, este trabajo muestra la disposición geográfica de la producción objetivo y la infraestructura disponible.

" Material Rodante: para un incremento de la carga como la proyectada, la disponibilidad de locomotoras y vagones es una limitante para aumentar la cantidad transportada. El incremento de su dotación es uno de los pilares para lograr el objetivo. Esta inversión se debe realizarse articulando al Estado y al sector privado (empresas de logística, o empresas cargadoras).

"Infraestructura Portuaria: la potenciación de los puertos de Barranqueras - Corrientes permitiría reducir la cantidad de granos que circulan por la vía terrestre (hoy transportados por modo automotor) y descongestionar los accesos de los puertos de Rosario. Además, las demoras que se generan por la falta de adecuados accesos a los puertos reducen la rotación de las formaciones y esta ineficiencia atenta contra la calidad y cantidad del servicio.

\section{Conclusión}

Este trabajo recurre a la idea de incrementar la competitividad argentina en la exportación de granos y subproductos introduciendo mejoras en la regulación, la infraestructura logística disponible en el país y la coordinación entre los agentes involucrados.

A lo largo del trabajo se ha localizado geográficamente la producción y procesamiento de los principales granos y derivados. Se ha concluido que la producción crece más velozmente que las zonas alejadas de los puertos, tanto en NEA y como en NOA.

Este fenómeno podría intuitivamente sugerir una mayor derivación a modos de transporte como el ferroviario y el fluvial, abaratando el costo medio. Para eso se ha identificado la infraestructura disponible y se mostró el recorrido de estas mercancías a lo largo del territorio del país.

Sin embargo, el transporte de granos por ferrocarril mostró un crecimiento lento que no solo no acompañó al veloz crecimiento de la producción de granos, sino que además empezó un proceso de decrecimiento a partir del 2012. Existieron además importantes disparidades según operador. 
Se presentó un indicador novedoso, el índice de penetración del ferrocarril en la carga de granos. Este indicador reflejó como el transporte por ferrocarril fue perdiendo relevancia en la producción agrícola del país.

Se analizaron las posibilidades económicas de trasladar carga según su localización y se sugirieron las principales obras de infraestructura para potenciar la misma y mejorar su logística. En línea con el postulado que indica que el ferrocarril y los barcos cargueros proveen un abaratamiento de costos medios y marginales en la medida que se trasladen grandes volúmenes en largas distancias.

Para el caso puntual de la región del NEA, principalmente la producción de la provincia del Chaco parece conveniente potenciar la carga fluvial en lugar que la ferroviaria, siendo indispensables obras en el puerto de Barranqueras.

Para el NOA, la existencia de una red ferroviaria en buenas condiciones y la ausencia de vías navegables, habilita a explorar alternativas para que la carga de la producción sea volcada en mayor medida a la red ferroviaria concesionada a NCA. Esta concesión ha mostrado un mejor desempeño en el transporte de granos que el resto de las concesiones.

Para la zona núcleo y sus alrededores, la red concesiona da FEPSA no ha podido acompañar con carga la mejora en la producción. Los granos y derivados de esta región recorren distancias cortas a los puertos y, por lo tanto, para competir económicamente con el transporte automotor es necesario pensar alternativas para manejar grandes volúmenes. Para esta concesión, estar integrada verticalmente con una empresa no vinculada a la explotación y/o industrialización agropecuaria, parece haber influido negativamente.

Desde el aspecto regulatorio y normativo se propone dotar al sistema ferroviario de mayores certezas, para garantizar que la migración de modo no generará dificultades operativas adicionales. 


\section{Q Bibliografía}

» Abad, J., Barbero, J. A. y Serebrisky, T. (2010). Logística. Análisis y Opciones para Resolver sus Desafíos Estratégicos. Banco Mundial. Disponible en: http://siteresources.worldbank.org/INTARGENTINAINSPANISH/Resources/ LogisticaArgentinall.pdf

»Barbero, J. A. (2007). Los ferrocarriles de cargas, la logística y el vínculo con la actividad portuaria. Banco Mundial. Disponible en: http://aapa.files.cms-plus. com/SeminarPresentations/o7_Rosario_Barbero_Jose.pdf

» Barbero, J. A. (2010). La logística de cargas en América Latina y el Caribe: una agenda para mejorar su desempeño. Banco Interamericano de Desarrollo (BID). № IDB-TN-103. Disponible en: https://publications.iadb. org/bitstream/handle/11319/6269/La\%2olog\%C3\%ADstica\%20de\%20 cargas\%20en\%20Am\%C3\%Agrica\%2oLatina\%20y\%20el\%2oCaribe\%3a\%20 una\%2oagenda\%2opara\%2omejorar\%20su\%2odesempe\% $\mathrm{C}_{3} \% \mathrm{~B}_{10}$. pdf?sequence $=1 \&$ isAllowed $=y$

»Barbero, J.A. (2014). Los Retos de la Logística para el Desarrollo Argentino. Instituto del Transporte. Universidad Nacional de San Martín. Disponible en: $\quad$ http://www.cera.org.ar/new-site/descargarArchivo.php?idioma_ code $=$ es\&contenido_id $=3372$

»Barbero, J. A. y Castro, L. (2013). Infraestructura logística. Hacia una matriz de cargas para la competitividad y el desarrollo sustentable. Centro de Implementación de Políticas Públicas para la Equidad y el Crecimiento (CIPPEC) Documento de Políticas Públicas №123. Disponible en: https://www. cippec.org/wp-content/uploads/2017/03/1396.pdf

»Calzada, J. y Rossi, G. (2015). ¿Cuál es el costo de eliminar los derechos de exportación al campo? Bolsa de Comercio de Rosario. Recuperado de: $\quad$ https://www.bcr.com.ar/Pages/Publicaciones/infoboletinsemanal. aspx?IdArticulo $=1278$

"Calzada, J. y Sese, A. (2017). Análisis del impacto de las Mejoras en Ferrocarriles sobre el Productor del Norte Argentino. Bolsa de Comercio de Rosario. Recuperado de: https://www.bcr.com.ar/Pages/Publicaciones/ informativosemanal_noticias.aspx?pldNoticia $=482$

"Canitrot, L. y García, N. (2012). La logística como herramienta para la competitividad. Cámara Argentina de la Construcción. Disponible en: http:// www.camarco.org.ar/File/GetPublicFile?id=1061

"Campos, G., De Gus, J. y Nombela, G. (2003). Economía del transporte. Universidad de las Palmas de Gran Canaria. Antoni Bosch Editores.

"Cayón, D. (2018, mayo 30). Para Bajar los Costos a Privados, Desregulan el Transporte Ferroviario de Cargas. El Cronista. Recuperado de: https://www. cronista.com/economiapolitica/Para-bajar-costos-a-privados-desregulan-eltransporte-ferroviario-de-cargas-20180530-0047.html

"López, P. (2018, septiembre 26). El campo espera un 2019 con cosecha récord y exportaciones por USD 26.000 millones. El Cronista. Recuperado de: https:// www.cronista.com/economiapolitica/El-campo-espera-un-2019-con-cosecharecord-y-exportaciones-por-us-26.00o-M-20180926-0046.html 
» Federman, D., Mangiameli, J. F. y Marcó, F. (2019). El desarrollo de las economías regionales. El caso del limón en Tucumán. En: Fernandez Bugna, C. y Gutti, P. (compiladores). Tras la búsqueda del desarrollo: planificación, financiamiento e infraestructuras en la Argentina (81-106). Buenos Aires: Universidad Nacional de Quilmes. Recuperado de: https://ridaa.unq. edu.ar/bitstream/handle/20.500.11807/1024/en_busca_del_desarrollo. pdf? sequence $=1$ \&isAllowed $=y$

"La Política Online (2018, mayo 29). Dietrich desregula el tren de carga. La Política Online. Recuperado de: http://www.lapoliticaonline.com/nota/113238dietrich-desregula-el-tren-de-carga/

"López, G. (2012). El Transporte De Granos En Argentina. Principal limitante del crecimiento del sector. Buenos Aires: Fundación Producir Conservando. Recuperado de: http://producirconservando.org.ar/intercambio/docs/trans.pdf

» Ministerio de Transporte (2017). Matrices de Orígenes y Destinos de Cargas. Buenos Aires: Ministerio de Transporte. Recuperado de: https://datos. transporte.gob.ar/dataset/informe-matriz-origen-destino-vial-de-transportede-cargas/archivo/b15197db-8b7e-4b78-87f7-1fdaeba47odd

»Muller, A. y Benassi, A. (2015). Un Modelo de Costos para el Transporte de Cargas en Argentina. Buenos Aires: Centro de Estudio de la Situación y Perspectivas de la Argentina (CEPES). Facultad de Ciencias Económicas de Universidad de Buenos Aires (FCE - UBA). Recuperado de: http://www.economicas.uba.ar/wpcontent/uploads/2015/11/41-Modelo-de-costos-red-bimodal-vial-ferroviariade-transporte-de_cargas_10Jun15_AB_o.pdf

"Raposo, I. (2013). Una Mirada Actual al Ferrocarril de Cargas en la Argentina. Buenos Aires: Revista Transporte y Territorio №10. Recuperado de: http:// revistascientificas.filo.uba.ar/index.php/rtt/article/view/520

»Secretaría de Agroindustria (2019). Datos Agroindustriales. Estimaciones Agrícolas. Secretaría de Agroindustria, Presidencia de la Nación. Recuperado de: https://datos.agroindustria.gob.ar/dataset/estimaciones-agricolas

»Sharp, R. (2005). Resultados de la Privatización de Ferrocarriles en América Latina. Washington: Banco Mundial. Recuperado de: http://siteresources.worldbank. org/INTTRANSPORT/Resources/336291-1227561426235/5611053-1229359963828/ TP-6_LAC_Railways_Concessions_spanish_web.pdf

\section{Normativa}

》Decreto 666/89 Recuperado de: http://servicios.infoleg.gob.ar/infolegInternet/ anexos/o-4999/1455/norma.htm

»Decreto 133/2015Recuperado de: http://servicios.infoleg.gob.ar/infolegInternet/ verNorma.do;jsessionid $=1368773150 \mathrm{~B}_{3} \mathrm{~F}_{35} \mathrm{BC}_{327} \mathrm{D}_{45} \mathrm{C}_{964} \mathrm{FC} 901$ id $=256979$

»Decreto 566/2013 Recuperado de: http://servicios.infoleg.gob.ar/infolegInternet/ anexos/21000o-214999/214826/texact.htm

»Decreto 1343/2016 Recuperado de: http://servicios.infoleg.gob.ar/infolegInternet/ anexos/27000o-274999/270114/norma.htm

»Decreto 793/2018 Recuperado de: http://servicios.infoleg.gob.ar/infolegInternet/ anexos/310000-314999/314042/texact.htm

"Ley 27.132 Recuperado de: http://servicios.infoleg.gob.ar/infoleglnternet/ anexos/24500o-249999/247081/norma.htm 


\section{Ezequiel Ayala / ezequielrayala@hotmail.com}

Ingeniero Aeronáutico egresado de la Universidad Tecnológica Nacional y maestrando de planificación y gestión del transporte (tesis pendiente) en la Universidad de Buenos Aires. Actualmente se desempeña como responsable del área de capacitación de la junta de investigación de accidentes de aviación civil y como docente en cursos de grado y posgrado.

\section{Darío N. Federman / dariofederman@hotmail.com}

Es Licenciado en Economía por la Universidad de Buenos Aires y master en Finanzas por la Universidad Torcuato Di Tella. Docente de Planificación del Desarrollo en la Universidad Nacional de Quilmes y en Administración Financiera (UNAJ-UNPAZ). Se desempeña como consultor en temas vinculados a la economía y las finanzas; análisis macro, sectorial, valuación de activos y de daños, tanto para el sector público como privado y organismos internacionales. Fue asesor del Directorio de la Comisión Nacional de Valores (2011-2014) y en la Dirección Nacional de Asuntos y Controversias Internacionales de la Procuración del Tesoro de la Nación (2012-2014).

\section{Juan F. Mangiameli / juanfmangiameli@gmail.com}

Licenciado en Ciencia Política con orientación en Administración Pública por la Universidad Nacional de Rosario. Máster en Economía y Desarrollo Industrial, Universidad Nacional General Sarmiento; Buenos Aires (en curso). Se desempeña como Director Nacional de Control de Gestión y Desarrollo en la Junta de Investigación de Accidentes de la Aviación Civil y como docente de Gestión de la Seguridad Operacional en la Universidad Provincial de Ezeiza. Tiene más de 15 años de experiencia en transporte.

\section{Federico Marcó / federicomarco.1@gmail.com}

Es Licenciado en Administración por la Universidad de Buenos Aires y especialista en docencia. Docente de materias sobre administración en UBA - UNAJ y UNPAZ. Fue asesor del Ministerio de Transporte de la Nación (2014-2015) y actualmente se desempeña dentro de la Secretaría de Políticas Universitarias. 